\title{
Stop-and-Go Algorithm for Blind Equalization in QAM Single-Carrier Coherent Optical Systems
}

\author{
Giulio Colavolpe, Member, IEEE, Tommaso Foggi, and Giancarlo Prati, Fellow, IEEE
}

\begin{abstract}
Coherent detection has recently allowed the adoption of high-order modulation formats in single-carrier optical systems where a simple feed-forward equalizer, in proper configuration, is able to perfectly compensate for fiber linear impairments, such as group velocity dispersion and polarization-mode dispersion. In this letter, the blind update of the equalizer taps is investigated with reference to a 16-ary quadrature amplitude modulation (QAM) format in the presence of different channel impairments. A novel algorithm is proposed, which represents an improvement of the stop-and-go, through the use of a powerful asynchronous detection strategy.
\end{abstract}

Index Terms-Equalization, quadrature amplitude modulation (QAM), signal processing.

\section{INTRODUCTION}

$\mathbf{T}$ HE recent and renewed diffusion of coherent detection in optical communications [1], [2] is pushed, besides other factors, by the need to increase the spectral efficiency, which can be reached through the exploitation of high-order modulation formats. Single-carrier polarization-multiplexed quadrature phase-shift keying (QPSK) is by now the best-established format for the upcoming 100-Gb/s systems [3]-[5]. Research is now addressing the $400-\mathrm{Gb} / \mathrm{s}$ or even $1-\mathrm{Tb} / \mathrm{s}$ systems [6], with a large variety of proposed solutions from orthogonal frequency-division multiplexing (OFDM) [7], to subcarrier multiplexing (SCM) [8], from novel modulation formats [9], to classical high-order modulations, like multilevel quadrature amplitude modulations (QAMs) [10], [11]. Predictably, the first efforts have been directed to the feasibility analysis of the wellknown QAM formats, which require a proper modulator, are more sensitive to phase noise and frequency offset, and, obviously, are less energy-efficient than simple QPSK schemes [12]. Besides these aspects, the convergence of blind strategies for the update of the equalizer taps requires to be investigated. In polarization-multiplexed coherent optical systems, it is known that linear equalization is sufficient to perfectly compensate for group velocity dispersion (GVD) and polarization-mode dispersion (PMD) (see [13] and references therein). If the convergence of the decision-directed least-mean-square (LMS) algorithm in blind mode (i.e., without training symbols) is not a concern with QPSK [2], [13], it is not the same with QAM formats. In the

Manuscript received August 20, 2010; revised September 20, 2010; accepted October 11, 2010. Date of publication October 21, 2010; date of current version December 02, 2010. This work was supported by Ericsson. A patent application was filed on September 2010.

G. Colavolpe and T. Foggi are with Dipartimento di Ingegneria dell'Informazione, University of Parma and CNIT Research Unit, I-43100 Parma, Italy. G. Prati is with Scuola Superiore Sant'Anna, I-56124 Pisa, Italy.

Digital Object Identifier 10.1109/LPT.2010.2089446

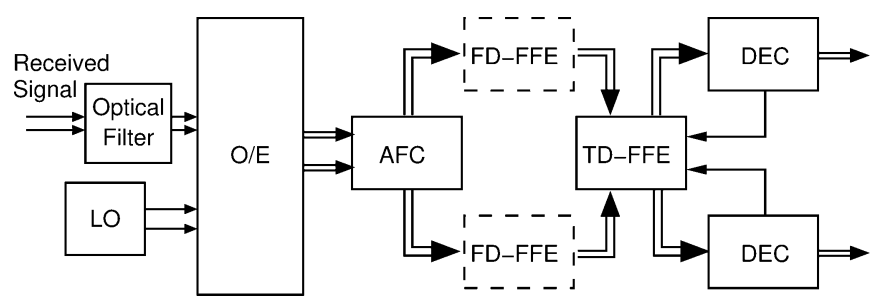

Fig. 1. Schematic of the proposed receiver.

literature, there exist several blind equalization algorithms for QAM [14]-[18]. In this letter, we compare some of them and also propose an enhancement of the algorithm in [19], to work jointly with the noncoherent detection strategy in [13], which was demonstrated to be a simple and effective technique to compensate for transmit and receive lasers' phase noise [13].

\section{SYSTEM MODEL}

The considered system model is detailed in [13]. Two 16-QAM symbol sequences $\left\{\mathbf{d}_{k}=\right.$ $\left.\left[d_{1, k}, d_{2, k}\right]^{T}, k=0,1, \ldots, K-1\right\},{ }^{1}$ obtained from symbols $\left\{\mathbf{a}_{k}=\left[a_{1, k}, a_{2, k}\right]^{T}, k=1,2, \ldots, K-1\right\}$, belonging to the same alphabet through quadrant differentially encoding [20], are linearly modulated with symbol time $T_{s}$ and launched on two orthogonal state of polarizations (SOPs) of a single-mode fiber (SMF). The channel introduces PMD and GVD. Optical amplification and filtering is performed at the receive end. Amplified spontaneous emission (ASE) noise is assumed to be dominant over thermal and shot noise. The ASE noise is modeled as additive white Gaussian noise (AWGN) whose complex envelope is composed by two independent complex noise components accounting for ASE on two orthogonal SOPs, each with two-sided power spectral density (PSD) equal to $N_{0}$. As shown in Fig. 1, an intradyne detection scheme is implemented (see [13] for details). Without loss of generality, we assume that two samples per symbol interval are extracted (the A/D resolution is assumed high enough to entail no penalty). A frequency offset compensation stage is then envisaged since the local oscillator is not locked to the incoming optical signal. The signal samples are then processed by an adaptive two-dimensional feed-forward equalizer (FFE). We assume that this equalizer is split into two fixed one-dimensional equalizers, one per polarization, implemented in the frequency domain and performing a rough GVD compensation, plus a short adaptive time-domain two-dimensional equalizer. We denote by $\mathbf{x}_{k}=\left[x_{1, k}, x_{2, k}\right]^{T}$ a column vector collecting the two-dimensional complex

\footnotetext{
${ }^{1}$ In the following, bold and upper-case-bold denote vectors and matrices, respectively, $(\cdot)^{T}$ denotes transpose, $(\cdot)^{*}$ complex conjugate, and $(\cdot)^{H}$ transpose conjugate.
} 
signal samples at the time instant $k T_{s},{ }^{2}$ one for each SOP, after the preliminary GVD compensation stage, and by $\mathbf{y}_{k}=\left[y_{1, k}, y_{2, k}\right]^{T}$ the equalizer output samples which are fed to the asynchronous symbol-by-symbol detection algorithm described in [13]. Output samples are related to input samples and equalizer taps as described in [13].

\section{Blind EQualization Algorithms}

As mentioned, amplitude/phase modulation formats like 16-QAM entail a troublesome convergence of the algorithms for the adaptation of the equalizer coefficients. In this work, a comparison between two different solutions is performed, chosen as the most effective for this scenario, namely the radius directed equalization (RDE) [17], [18] and the stop-and-go (SG) [19] algorithms. The RDE is implemented combined with the carrier phase recovery algorithm described in [18], and it is taken as a reference for its good performance. The SG is modified with respect to [19], since the presence of the asynchronous detection strategy in [13], used for both detection and equalizer tap update, is exploited. This new algorithm will be called asynchronous stop-and-go $(a-\mathrm{SG})$ and the corresponding error signal reads

$$
\hat{\mathbf{e}}_{k}=\left[\hat{e}_{1, k}, \hat{e}_{2, k}\right]^{T}=\left(\mathbf{y}_{k}-\mathbf{g}_{k} \odot \hat{\mathbf{d}}_{k}\right)
$$

where $\hat{\mathbf{d}}_{k}=\left[\hat{d}_{1, k}, \hat{d}_{2, k}\right]$ are the decisions, $\odot$ denotes the Hadamard (entrywise) product, and $\mathbf{g}_{k}=\left[g_{1, k}, g_{2, k}\right]^{T}$, where

$$
g_{i, k}=\frac{\sum_{n=1}^{N_{p}} y_{i, k-n} \hat{d}_{i, k-n}^{*}}{\left|\sum_{n=1}^{N_{p}} y_{i, k-n} \hat{d}_{i, k-n}^{*}\right|} .
$$

The two-dimensional equalizer coefficients at discrete time $k$ are represented through complex $2 \times 2$ matrices $\left\{\mathbf{C}_{\ell}^{(k)}\right\}, \ell$ ranging over the equalizer length [13].

As in the original SG algorithm, the coefficient update is modified with the introduction of two flags $\mathbf{f}_{k}=\left[f_{1, k}, f_{2, k}\right]^{T}$ :

$\hat{\mathbf{C}}_{\ell, R}^{(k+1)}=\hat{\mathbf{C}}_{\ell, R}^{(k)}-\alpha\left(\mathbf{f}_{k, R} \odot \hat{\mathbf{e}}_{k, R} \mathbf{x}_{k-\ell, R}^{H}+\mathbf{f}_{k, I} \odot \hat{\mathbf{e}}_{k, I} \mathbf{x}_{k-\ell, I}^{H}\right)$ $\hat{\mathbf{C}}_{\ell, I}^{(k+1)}=\hat{\mathbf{C}}_{\ell, I}^{(k)}+\alpha\left(\mathbf{f}_{k, R} \odot \hat{\mathbf{e}}_{k, R} \mathbf{x}_{k-\ell, I}^{H}-\mathbf{f}_{k, I} \odot \hat{\mathbf{e}}_{k, I} \mathbf{x}_{k-\ell, R}^{H}\right)$

where $\alpha$ is the step size and $\{R, I\}$ denote real and imaginary components. The flags $f_{i, k,\{R, I\}}$ are set equal to 1 (otherwise to 0$)$ only if $\operatorname{sgn}\left(\hat{e}_{i, k,\{R, I\}}\right)=\operatorname{sgn}\left(\tilde{e}_{i, k,\{R, I\}}\right)$, where $\tilde{e}_{i, k,\{R, I\}}=y_{i, k,\{R, I\}}-\operatorname{sgn}\left(y_{i, k,\{R, I\}}\right) \beta_{k}$, with the value of $\beta_{k}$ properly chosen (possibly time-varying) [19]. In this way, a novel algorithm results, combining the effectiveness of the SG blind equalization with the robustness of the asynchronous detection strategy.

\section{NUMERICAL RESULTS}

In Table I, three different channels are described, in terms of GVD (here expressed through the dimensionless parameter $\gamma=$ $2 \lambda_{0} R_{s}^{2} D_{r} / \omega_{0}$, where $\lambda_{0}$ and $\omega_{0}$ are the optical carrier wavelength and angular frequency, respectively, $R_{s}$ is the symbol

\footnotetext{
${ }^{2} \mathrm{As}$ mentioned, two samples per symbol interval $T_{s}$ fed the equalizer, at time $k T_{s}$ and at time $k T_{s}+T_{s} / 2$. However, only that at time $k T_{s}$ is employed for the adjustment of the equalizer's coefficients [13].
}

TABLE I

GVD AND PMD PARAMETERS FOR THE THREE CONSIDERED CHANNELS

\begin{tabular}{|l|c|c|c|c|c|}
\hline & $\gamma$ & $\tau / T_{s}$ & $\tau_{\omega} / T_{s}^{2}$ & $q_{\omega} / T_{s}$ & $\rho$ \\
\hline Ch. 1 & 0.8 & 0.6 & 0.2 & 0.3 & 0.7 \\
\hline Ch. 2 & 1.6 & 1.8 & 1.0 & 0.6 & 0.7 \\
\hline Ch. 3 & 2.0 & 1.3 & 0.5 & 0.5 & 0.4 \\
\hline
\end{tabular}

TABLE II

BER AND CONVERGENCE TIME MEASURED IN SYMBOL INTERVALS FOR THE $a$-SG AND RDE ALGORITHMS, FOR THE THREE CONSIDERED CHANNELS AT $E_{b} / N_{0}=12 \mathrm{~dB}$

\begin{tabular}{|c|c|c|c|c|c|c|}
\hline & \multicolumn{2}{|c|}{ Ch. 1 } & \multicolumn{2}{c|}{ Ch. 2 } & \multicolumn{2}{c|}{ Ch. 3 } \\
\hline & RDE & $a$-SG & RDE & $a$-SG & RDE & $a$-SG \\
\hline \hline BERx10 $^{-2}$ & 0.626 & 0.580 & 1.99 & 1.29 & 1.89 & 1.24 \\
\hline$T_{\text {conv }}$ & 36400 & 17900 & 73100 & 26300 & 75300 & 26900 \\
\hline
\end{tabular}

rate, and $D_{r}$ the residual dispersion) and second-order PMD parameters, all normalized to the symbol time $T_{s}=1 / R_{s}$ (differential group delay $\tau$, its derivative $\tau_{\omega}$, rotation rate $q_{\omega}$, power splitting $\rho$ ). An arbitrary polarization rotation is also considered in each case. In all cases, it is $N_{p}=8, \beta_{k}=0.65$ (independent of $k$ ), and the step size of all algorithms has been optimized through computer simulations (turning out to be almost always about $10^{-2}$ in these cases, given the QAM constellation normalized to unit power). In case of channel \#1, the number of equalizer taps is equal to 15 , whereas for channels \#2 and \#3, the number of equalizer taps has been increased to 27 and 31 , respectively. A fourth-order Gaussian optical filter with bandwidth equal to $0.9 / T_{s}$ is present both at transmit and receive ends.

Given these channel and receiver configurations, we measured the convergence time and the bit-error rate (BER). Convergence time $T_{\text {conv }}$, measured in symbol intervals, is triggered when the algorithm error signal falls below the $120 \%$ of its steady-state value. The initial equalizer coefficients $\left\{\mathbf{C}_{\ell}^{(k)}\right\}$ are all set to zero except for the central one, set to the $2 \times 2$ identity matrix. Results averaged over ten trials for each channel are reported in Table II. It can be noticed that the $a$-SG algorithm is more than twice as fast as the RDE with a smaller BER. Moreover, the RDE algorithm locks its outputs on the same polarization much more frequently than the $a$-SG algorithm (although in both cases this problem can be avoided by using the technique in [21]). These wrong locks were not considered in the results of Table II. From Fig. 2, where the mean-square-error (MSE) curves are shown, it is possible to verify that the $a$-SG algorithm is faster than RDE to converge to a lower steady-state MSE.

To highlight the greater robustness of the $a$-SG algorithm, we now consider channel \#2 in the presence of phase noise (modeled as a Wiener process, i.e., a process with i.i.d. Gaussian increments, whose standard deviation depends on the laser linewidth $\Delta \nu$ ) and an uncompensated frequency offset $F_{0}$. We only consider the effect of the receive phase noise (the transmit phase noise is neglected) assuming that the normalized laser linewidth is $\Delta \nu T_{s}=10^{-4}$ and that the normalized uncompensated frequency offset is $F_{0} T_{s}=10^{-3}$. The BER corresponding to both RDE and $a$-SG algorithms is shown in Fig. 3 along with the back-to-back BER reported for comparison. As can be observed, the $a$-SG algorithm performs better although a BER floor is reached, due to the presence of the strong phase noise. 

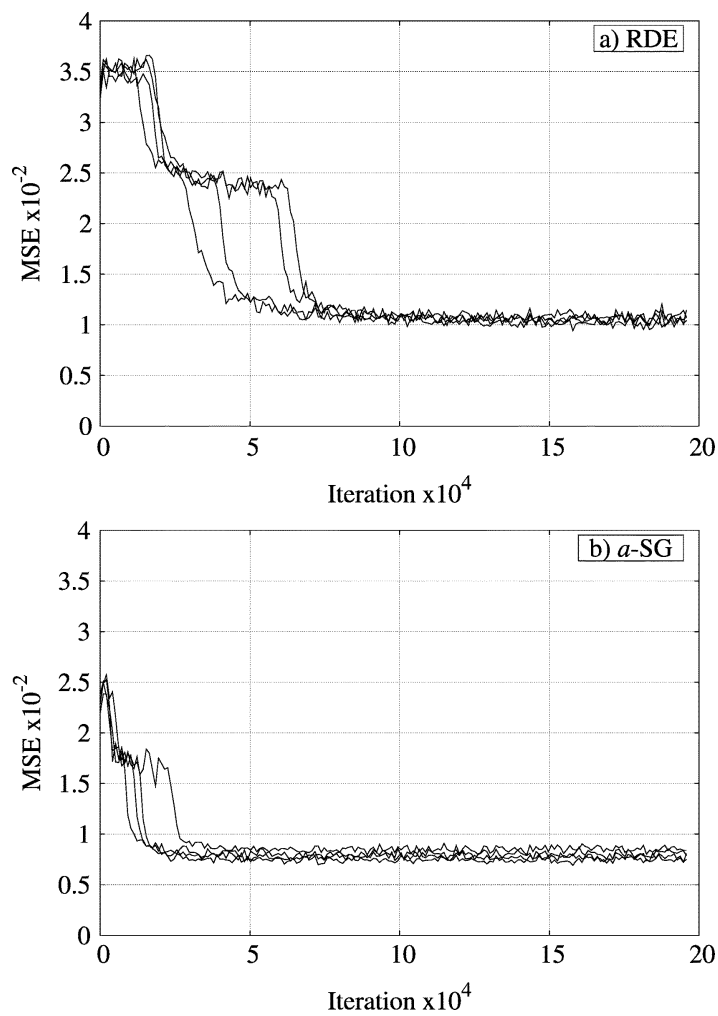

Fig. 2. MSE of the two compared algorithms, (a) RDE and (b) $a$-SG, for several trials over channel \#2, with $E_{b} / N_{0}$ equal to $12 \mathrm{~dB}$. The steady state is reached much faster with the $a$-SG. The MSE was computed averaging over a sliding window of $N=1000$ symbols, i.e., denoting by $\mathrm{MSE}_{k}$ the MSE at discrete time $k$, it is $\mathrm{MSE}_{k}=1 / N \sum_{\ell=0}^{N-1}\left\|\mathbf{e}_{k}\right\|^{2}$, where $\mathbf{e}_{k}$ has the same definition of $\hat{\mathbf{e}}_{k}$ but with transmitted data instead of decisions.

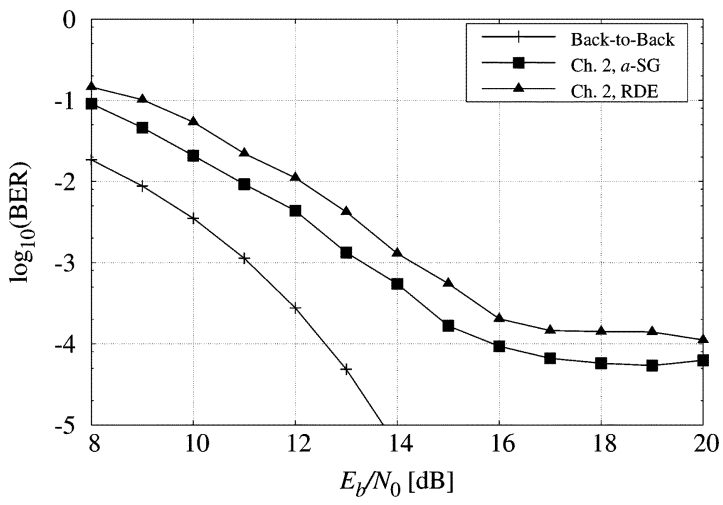

Fig. 3. BER curves of the back-to-back ideal case and of the two compared algorithms ( $a$-SG and RDE) for channel \#2 and in the presence of phase noise $\left(\Delta \nu T_{s}=10^{-4}\right)$ and an uncompensated frequency offset $\left(F_{0} T_{S}=10^{-3}\right)$. The receiver parameters are optimized through computer simulations.

\section{CONCLUSION}

We compared two blind equalization algorithms for 16-QAM coherent optical systems, namely the radius directed equalization algorithm and the stop-and-go algorithm, properly modified to increase its robustness against phase noise. Results show that the new algorithm is fast and reliable, as it performs better than RDE both in terms of convergence time and steady-state performance.

\section{REFERENCES}

[1] G. Raybon and P. J. Winzer, " $100 \mathrm{~Gb} / \mathrm{s}$ challenges and solutions," in Proc. Optical Fiber Commun. Conf. (OFC'08), San Diego, CA, Feb. 2008, Paper OTuG1.

[2] E. M. Ip and J. M. Kahn, "Fiber impairment compensation using coherent detection and digital signal processing," J. Lightw. Technol., vol. 28, no. 4, pp. 502-519, Feb. 15, 2010.

[3] P. J. Winzer, G. Raybon, H. Song, A. Adamiecki, S. Corteselli, A. H. Gnauck, D. A. Fishman, C. R. Doerr, S. Chandrasekhar, L. L. Buhl, T. J. Xia, G. Wellbrock, W. Lee, B. Basch, K. Kawanishi, T. Higuma, and Y. Painchaud, "100-Gb/s DQPSK transmission: From laboratory experiments to field trials," J. Lightw. Technol., vol. 26, no. 20, pp. 3388-3402, Oct. 1, 2008.

[4] G. Charlet, M. Salsi, P. Tran, M. Bertolini, H. Mardoyan, J. Renaudier, B.-P. O. , and S. Bigo, "72 $\times 100 \mathrm{~Gb} / \mathrm{s}$ transmission over transoceanic distance, using large effective area fiber, hybrid Raman-Erbium amplification and coherent detection," in Proc. Optical Fiber Commun. Conf. (OFC'09), San Diego, CA, Mar. 2009, Paper PDPB6.

[5] A. Barbieri, G. Colavolpe, T. Foggi, E. Forestieri, and G. Prati, "OFDM versus single-carrier transmission for $100 \mathrm{Gbps}$ optical communication," J. Lightw. Technol., vol. 28, no. 17, pp. 2537-2551, Sep. 1, 2010.

[6] T. Hirooka, M. Nakazawa, M. Yoshida, K. Kasai, P. Guan, and T. Hirano, "Recent progress on OTDM terabit/s transmission and their future," in Proc. Optical Fiber Commun. Conf. (OFC'10), San Diego, CA, Mar. 2010, Paper OWM5.

[7] S. L. Jansen, I. Morita, T. C. W. Schenk, N. Takeda, and H. Tanaka, "Coherent optical 25.8-Gb/s OFDM transmission over 4160-km SSMF," J. Lightw. Technol., vol. 26, no. 1, pp. 6-15, Jan. 1, 2008.

[8] A. Sano, E. Yamada, H. Masuda, E. Yamazaki, T. Kobayashi, E. Yoshida, Y. Miyamoto, R. Kudo, K. Ishihara, and Y. Takatori, "No-guard-interval coherent optical OFDM for 100-Gb/s long-haul WDM transmission," J. Lightw. Technol., vol. 27, no. 16, pp. 3705-3713, Aug. 15, 2009.

[9] E. Agrell and M. Karlsson, "Power-efficient modulation formats in coherent transmission systems," J. Lightw. Technol., vol. 27, no. 22, pp. 5115-5126, Nov. 15, 2009.

[10] P. J. Winzer, A. H. Gnauck, C. R. Doerr, M. Magarini, and L. L. Buhl, "Spectrally efficient long-haul optical networking using $112-\mathrm{Gb} / \mathrm{s}$ polarization-multiplexed 16-QAM," J. Lightw. Technol., vol. 28, no. 4, pp. 547-556, Feb. 15, 2010.

[11] M. Nakazawa, S. Okamoto, T. Omiya, K. Kasai, and M. Yoshida, "256-QAM (64 Gb/s) coherent optical transmission over $160 \mathrm{~km}$ with an optical bandwidth of $5.4 \mathrm{GHz}$, , J. Lightw. Technol., vol. 22, no. 3, pp. 185-187, Feb. 1, 2010.

[12] J. G. Proakis, Digital Communications, 4th ed. New York: McGrawHill, 2001.

[13] G. Colavolpe, T. Foggi, E. Forestieri, and G. Prati, "Robust multilevel coherent optical systems with linear processing at the receiver," J. Lightw. Technol., vol. 27, no. 13, pp. 2357-2369, Jul. 1, 2009.

[14] Y. Sato, "A method of self-recovering equalization for multilevel amplitude-modulation systems," IEEE Trans. Commun., vol. COM-23, no. 6, pp. 679-682, Jun. 1975.

[15] D. N. Godard, "Self-recovering equalization and carrier tracking in two-dimensional data communication systems," IEEE Trans. Commun., vol. COM-28, no. 11, pp. 1867-1875, Nov. 1980.

[16] A. Benveniste and M. Goursat, "Blind equalizers," IEEE Trans. Commun., vol. COM-32, no. 8, pp. 871-883, Aug. 1984.

[17] J. Yang, J.-J. Werner, and G. A. Dumont, "The multimodulus blind equalization and its generalized algorithms," IEEE J. Sel. Areas Commun., vol. 20, no. 3, pp. 997-1015, Jun. 2002.

[18] I. Fatadin, D. Ives, and S. J. Savory, "Blind equalization and carrier phase recovery in a 16-QAM optical coherent system," J. Lightw. Technol., vol. 27, no. 15, pp. 3042-3049, Aug. 1, 2009.

[19] G. Picchi and G. Prati, "Blind equalization and carrier recovery using a 'stop-and-go' decision directed algorithm," IEEE Trans. Commun., vol. 35, no. 9, pp. 877-887, Sep. 1987.

[20] W. J. Weber, "Differential encoding for multiple amplitude and phase shift keying systems," IEEE Trans. Commun., vol. 26, no. 3, pp. 385-391, Mar. 1978.

[21] X. Liu, F. Buchali, and R. W. Tkach, "Improving the nonlinear tolerance of polarization-division-multiplexed CO-OFDM in long-haul fiber transmission," J. Lightw. Technol., vol. 27, no. 16, pp. 3632-3640, Aug. 15, 2009. 